\title{
Emergency Management for Disasters in Malaysian Hotel Industry
}

\author{
Ahmad Rasmi AlBattat ${ }^{1}$, Ahmad Puad Mat Som², Ala a Abukhalifeh ${ }^{3}$ \\ ${ }^{1,2,3}$ School of Housing, Building and Planning, Universiti Sains Malaysia, Penang, Malaysia \\ ${ }^{1,2}$ Sustainable Tourism Research Cluster, Universiti Sains Malaysia, Penang, Malaysia
}

\begin{abstract}
This paper aims to identify major emergencies that have the potential to place Malaysian hotels in emergency and disaster situations; investigate how hotels were prepared for emergencies, how they manage and overcome emergencies when occurred; and limitations and factors influencing successful emergency planning and adoption emergency management in Malaysian hotels. Face-to-face interview with managers from three, four and five star hotels from different backgrounds: local; regional; and International in Kuala Lumpur, Subang, and Putrajaya are undertaken. The result revealed that Malaysian hotels are exposed to a wide range of natural and man-made disasters. Malaysian hotels lack proactive emergency planning and a lot of constraints which impede successful emergency planning for disasters in the hotel industry in Malaysia, with emphasizing on the relevant authority's role to demonstrate emergency management to hotels convincing them to adopt such practices, so they can be able to cope with emergencies effectively.
\end{abstract}

\section{Introduction}

Since the last few decades, the hospitality industry in the world generally and in Southeast Asia, particularly, has been subjected to several disasters and emergencies affecting the inbound arrivals and tourist revenue, creating multiple challenges for the private and public sectors [1]. A wave of catastrophic events, such as the September 11 attacks on the World Trade Center in New York, terrorist attacks in Bali, Southeast Asia Tsunami, Arab spring revolution, have increased awareness among hospitality scholars, raising questions about how to read, manage and mitigate the effects of such events on the hospitality sector [2]. Other scholars identified the major failures when dealing with disasters and hazardous events [3]. Scholars explain how hospitality organizations could benefit from applying emergency management to prevent losses, and the important role of media to enhance the destination image [4].

Disaster has been defined as "an emergency situation of some complexity that will cause the loss of lives, damage of property and the environment, and harm local social and economic activities" [5]. Other scholars define it as "an extreme event with a natural, technological or social cause that has consequences in term of casualties, destruction, damage and disruption" [6]. Casualties which have a broader term that include disasters, catastrophes, and disruptive events has been defined as "an imminent or actual event that threatens people, property or the environment and which requires a 
coordination and rapid response" [6]. These emergency situations have been classified into natural and man-made disasters. The catastrophic events caused by nature, such as floods, tsunamis, typhoons, etc. are called natural disasters, and can't be controlled by humans, and often termed "Act of God" [7]. Henderson [8] asserted that man-made disasters are known as socio-technical disasters and could be occurred in four types: technical disasters, transport failure, stadia or "public place" failures, and production failures.

This research investigates the impact of current disasters on Malaysian hotel industry as one of the most popular destinations in Southeast Asia. Semi-structured interviews were conducted with hotel managers in Malaysia, using a purposeful snowball method. Questions were asked about the current disasters affecting hospitality and tourism industry in Malaysia, and the most effective strategies for coping with such hazardous events. In spite of several researches on disaster and emergency management in hospitality and tourism studies, this field is relatively new and more studies should be done to investigate the impact of disasters in Southeast Asia as a whole and Malaysia in particular. After a brief introduction of the study, this research will review related literature, present the study findings, and then highlight the main points in the conclusion section.

\section{$2 \quad$ Literature Review}

\subsection{Emergency Planning and Preparedness}

The last few decades have witnessed a considerable increment in the number, scope and complexity of disasters and emergencies. Planning is a process; created plans should be tested and evaluated through practice. Helsloot and Ruitenberg [9] explain that the difference between planning and preparedness comes from the practice. Planning should be the standard of preparedness. [10] defined the emergency plan as "a coordinated set of protocols for managing an adverse event, whether expected or untoward, in the future". Every emergency is unique, but with enough common ground between them it will be easier to make predictions, forecasting, warning, and planning [11]. Therefore, it is very important to attract more attention to emergency preparedness, and the planning process to manage, overcome and recover emergencies [11]. Emergency planning, response, and recovery can be a great challenge to any business, especially hospitality industry regarding to the worse situation from experiencing an emergency event far from home. In a stricken destination, tourists are coming from anywhere on earth and speak different languages [12]. [13] argued that a lot of investigations should be done to determine the best disaster management model, he mentioned that planning is not managing disasters, and future disasters are not a repeat of the past.

The quality of emergency planning should be monitored, evaluated, and improved for several reasons. First, emergency management is not yet a fully-fledged profession [14], with a lack of adequate training and specialist knowledge for emergency planners. Second, inefficiency in emergency planning which raises the mismatches between the procedures and available resources in balance with chronic emergency needs. Third, emergency planning should be a dynamic continuous process, since it's become static it will be mutated to become dysfunctional [15]. [5] argued that emergency planning is not a continuous process and to be regarded to be ended by itself. Alexander [11] defends this statement and argued that it should be dynamically processed to insure functionality. Planning by hotels should be in collaboration with local authorities to bridge the gap between internal and external plans [12]. Fourth, informal planning which contains vague definition of responsibilities and procedures, and poorly indicated [16]. Finally, emergency plans shouldn't be isolated from presiding laws and regulations. Scholars have noted that emergency planning should in a multi organizational nature [15]. 


\subsection{Disaster and emergency management}

[17] define a disaster as an unusual event which causes damage, loss, and injury. These events overwhelm the capacity of organizations and require recovery efforts in order to return back to normal situation [18]. Emergency management for disasters can be a great challenge to any business, especially hospitality industry regarding the bad situation from experiencing an emergency event far from home [19]. Scholars have argued that emergency managers should determine the best model or methodology when preparing for, reacting to, and recover from an emergency situation. Quarantelli [20] mentioned in his continuing research that planning is not managing disasters, and future disasters are not a repeat of the past. [21] examined the level of disaster preparedness and evacuation planning for tourist businesses to determine the impact of planning on preparedness, forces, and lesson learnt such as action plans, who's in charge, and communication.

The quality of emergency planning should be monitored, evaluated, and improved for several reasons. First, emergency management is not yet a fully-fledged profession [14], with a lack of adequate training and specialist knowledge for emergency planners. Second, inefficiency in emergency planning raises the mismatches between the procedures and available resources in balance with chronic emergency needs. Third, emergency planning should be a dynamic continuous process, since it's become static it will be mutated to become dysfunctional [15]. [5] argued that emergency planning is not a continuous process and to be regarded to be ended by itself. Alexander [11] defends this statement and argued that it should be dynamically processed to insure functionality. Planning by hotels should be in collaboration with local authorities to bridge the gap between internal and external plans [12]. Fourth, informal planning which contains vague definition of responsibilities and procedures, and poorly indicated [16]. Finally, emergency plans shouldn't be isolated from presiding laws and regulations. Scholars have noted that emergency planning should in a multi organizational nature [15]. Good plans and teams are essential requirements for surviving from disasters. Hard work and many difficult decisions are very important in the case of emergency recovery. From the end of post-emergency period until the reestablishment of the trend line emergency recovery involves all effort to deal, manage and recover from the disastrous situation.

In the wake of disaster, resource management and allocation is essential regarding the challenges facing the hotels in the pre-during-post emergency based on flattening organization structure, team dealing with emergencies [22]. Scholars have proposed different models to deal with disasters and emergencies, and prevent hotels from its impacts. According to [23] disaster management model, emergency management should start before the disaster happens and start affects the hotel industry. Emergency management could be divided into four stages: Prodormal, Acute, Chronic, and resolution. $\mathrm{He}$ asserted that early warning signals for even repeated disasters are difficult to recognize. Moving from prodormal to acute stage, disasters start to cause damage and losses, the preparedness level and the effectiveness dealing with emergencies could be contributed to losses degree. In contrast, the chronic stage allows the organization to recover from the disaster and learn from the strengths and weaknesses in emergency response plan. In his model, [24] explained four stages of disaster management. The pre-event stage where the efforts to mitigate the impact of and to be prepared for the potential disaster, in the emergency stage disaster happens and actions will be taken to rescue and save people and properties, in the intermediate stage hotels provides short-term plans to restore essential services and overcome as quickly as possible, and finally, long-term stage is where the repair of infrastructure using the long term strategies, and enhance the emergency plans to prepare for the next emergency.

Faulkner disaster management framework considered as one of the most effective models to deal with emergencies. [25] identified six phases in his model: pre-event; prodormal; emergency; intermediate; long-term; and resolution. In the pre-even phase hotels have enough time to prevent and mitigate emergencies. The prodormal phase where disaster avoidance is no longer possible, and the necessary need to activate emergency plans. In the emergency phase all efforts to save lives and properties. In the intermediate phase the primitive need for help and evacuate injured people. In the long-term phase, the efforts focus on the preparedness management to identify and improve 
weaknesses and bridging the gaps to learn from disaster. Finally, in the resolution phase hotels learnt the lesson and improve their plans to be prepared for the next disaster.

\section{$3 \quad$ Methodology}

This study aims to investigate the effect of disasters and emergencies on hospitality industry in Malaysia, emergency planning and management. To undertake this study, a qualitative research methodology was considered the most appropriate method to describe the existing issues related to disasters and emergencies affecting Malaysia. Secondary information was assembled from governmental resources, while in the qualitative approach, semi-structured face-to-face interviews have been used [26]. The use of semi-structured interviews conducted in June 2012, with a number of pre-determined questions, facilitated the scientific comparison between the responses of participating interviewees [27]. 33 key persons in charge of Malaysian hospitality industry were interviewed [28]; this sample was selected using the purposeful snowball method. Regarding this method, personal links were used to identify the most knowledgeable persons. The sample size was determined using the 'saturation criterion'. The senior managers were drawn from the hospitality organizations in Malaysia, namely, Small local, medium regional and large international hotels. The materials collected in this study were subjected to qualitative thematic analysis aiming to uncover the prevalent themes by identifying, describing and reporting the content of data. Results will be outlined next, featuring direct quotations to support the study's findings.

\section{$4 \quad$ Findings and Discussion}

This research was carried out among a number of Malaysian Hospitality key players in Federal Territories of Kuala Lumpur, Putrajaya, and Selangor, Pulau Penang, Kedah (Langkawi). To investigate a variety of issues related to emergency management for disaster in the Malaysian hospitality industry. Issues however explored type of disasters, emergency planning, and strategies for response, and recommendations from the interviewees.

\subsection{Typology and Impact of Disasters}

Malaysia was not immune from several types of disasters affecting the hospitality industry in Southeast Asia and the rest of the world. Respondents admitted that the hospitality sector in Malaysia had been affected by several local, regional, and global disasters. Disasters classified as diseases, terrorism, natural disasters, and economic. Arising broadly outside Malaysia, these disasters were an issue of deep concern to the hospitality industry, there were differences among respondents in perceived impact, depending upon the scale, size of business, and stricken destinations. Each disaster had its own characteristics, during the outbreak of SARS, hospitality industry received a formidable shock and international arrivals reduced by $20 \%$ in some states. Approximately, all respondents emphasized that SARS and Avian flu created intricate situation and "it took us more than 6 months to recover". In line with epidemics, tourist arrivals fluctuated in Malaysia and surrounded region regarding the first Bali bombing in 2002, and the second Bali bombing 2005. "Though it occurred in Indonesia but it affects Malaysian hospitality industry" regarding to the opinion of Malaysian key players. Furthermore, the bad impact of media, which mention that the ripple impact was happening in Malaysia which pointed out in an article published in the Asian Wall Street Journal, The matter which forces the government to "spend a lot of money" to market Malaysia as a terrorist-free destination.

According to respondents, the deep impact of the Asian tsunami caused losses estimated at around RM30 million (US\$8 million) in the northern affected states of Perak, Perlis, Penang and Kedah. Some hotels and beach resorts suffered a lot from dropping tourist arrivals for a short period of time. The hospitality industry also has been affected by the global economic crisis and the outbreak of H1N1 in 2008 and 2009. The key respondents claimed that the severe impact was due to reservation 
cancellation, reducing the length of stay with less expenditure from tourists. This situation affects not only hotels but all key players in tourism business, airlines, and tour operators. Senior managers emphasized that dramatic drop in hotel occupancy rates has been experienced, With less spending on leisure, shopping, food and beverage, especially SMEs who clarifies that they were "unable to cope with disaster without financial support". Hospitality industry also has been suffering from "the drop in tour packages" from the western markets from USA and Canada, Scandinavian countries, west and central Europe, Australia and New Zealand as respondent answers.

\subsection{Emergency Management}

Respondents also were asked how they managed and recovered from these disasters, and how they used emergency planning to return back to normality. Respondents asserted that the government introduced marketing campaigns to promote Malaysia like "Cuti Cuti Malaysia" to convince domestic tourists. Also, the government start to give the citizen paid leave if they want to travel domestically. Furthermore, produce different tourism product like agro-tourism, rural tourism, and home stay. Hotels also start to attract domestic tourists by providing massive discounts, especially in low seasons, in the weekdays, and during disasters to compensate the shortage of outbound tourism. The majority of respondents agreed that marketing campaign strategies undertaken have a very strong influencing others to visit Malaysia such as "Visit Malaysia year", "Malaysia truly Asia", and "Malaysia my second home". This publicity fosters the recovery process, and gives the confidence to the tourist to return back to Malaysia. Other strategies have been used to recover from the disasters such as increasing the service quality, hotel renovation, tax reduction, and cheap tour packages. Confirmed by respondents from industry who stated that marketing recovery campaign`s efforts had made a difference, and was very important to sustain the business. Results suggest that the majority of respondents support the government marketing campaigns following disasters.

Respondents from local and regional hotels confessed that they don't have any emergency planning for disasters. They argued that they implemented some recovery strategies in the past with no impact, as they overshadowed by the negative impact of disasters especially with the negative role of media coverage. Respondents who don't have proactive planning and recovery strategies reported that they don't want to do such kind of planning if the disaster will occur again, and conclude that "we don't have sufficient resources", "our work is too small" and "what we can do in the face of disasters". While, other respondents from International hotels reported that they have emergency planning for disasters and hazardous events. Few of them have formal written plans, and other noted that they develop non documented plans. This will give an indication that bottom-up to emergency proactive planning is not effective, since some respondents seem unwilling to develop their own proactive plans. Further questions to the respondents about the contents of their written plans, around half of them report that their plans are "general" or merely "fire and evacuation plans". And a quarter of the respondents listed two or more emergency planning scenarios to deal with emergencies and recover from disasters.

Respondents from government agencies and International hotels mentioned that information and experience from disasters would increase emergency planning and recovery strategies will increase awareness of the potential threats, and give a better understanding of the role of government and other organizations in the three stages of disaster; before a disaster (proactive emergency planning), during a disaster (response), and after a disaster (recovery). While, small and medium business claims that they don't have this kind of planning regarding "lack of money", "lack of education and awareness", and "the nature of their business". This will give an indication that although disasters affected hospitality in Malaysia, respondents in the case of disasters acted based an ad hoc manner with no priority for emergency planning in the industry. 


\section{Conclusion}

Malaysia has subsequently been struck by a range of global and regional disasters. Reflecting the hospitality industry vulnerability to hazardous events in the internal and external environment, they have caused dramatic fluctuations in Tourist arrivals and revenue, and reduce occupancy rate for hotels in certain periods. Events discussed in this research disclose a wave of disaster and emergencies affecting the hospitality industry in Malaysia over the last few decades, which affects this industry's contribution to Malaysian GDP and reveals the multiplier effect on the economy. The finding emphasizes the power of media in clearing the ambiguity, which helps marketing efforts towards tourists to change their attitudes and visit Malaysia rather than visiting hazardous destinations. Proactive emergency planning and the urgent need for better cooperation, coordination and awareness efforts among the industry stakeholders, government agencies, and local communities could mitigate the negative impact of hazardous events, allowing organizations to learn from the past to prevent the same effects in the future. Increase awareness, enhance services, secure sufficient fund, emergency planning knowledge will help the industry to be well prepared for future disasters.

\section{$6 \quad$ Acknowledgments}

This research was fully funded from the Sustainable Tourism Research Cluster, Universiti Sains Malaysia, [Grant No. 1001/PTS/8660013] 'Tourism Planning'.

\section{References}

1. B. Prideaux. The Need to Use Disaster Planning Frameworks to Respond to Major Tourism Disasters. Journal of Travel \& Tourism Marketing, 15,4, p. $281-298$ (2004)

2. E. Cohen. Explorations in Thai tourism: Collected case studies. 11: Emerald Group Publishing. (2008)

3. A. Gheytanchi, et al., The dirty dozen: Twelve failures of the Hurricane Katrina response and how psychology can help. American Psychologist, 62: p. 118-130 (2007)

4. W.R. Kerr, Chapter 1 - Aspects of the Tourism Industry in the 21st Century, in Tourism Public Policy, and the Strategic Management of Failure, W.R. Kerr, Editor. Pergamon: Oxford. 321(2003)

5. H.I. Kuo et al., Assessing impacts of SARS and Avian Flu on international tourism demand to Asia. Tourism Management, 29,5, 917-928 (2008)

6. R. Perry and E. Quarantelly. what is Disaster? New Answers to Old Questions. Xlibris Press, Philadelphia, PA (2004)

7. A. Wilder-Smith, The severe acute respiratory syndrome: impact on travel and tourism. Travel medicine and infectious disease, 4,2, 53-60 (2006)

8. J. Henderson. Corporate social responsibility and tourism: hotel companies in Phuket, Thailand, after the Indian Ocean tsunami. International Journal of Hospitality Management, 26,1, 228-239(2007)

9. I. Helsloot and A. Ruitenberg, Citizen response to disasters: a survey of literature and some practical implications. Journal of Contingencies and Crisis Management, 12,3, 98-111 (2004)

10. D. Alexander. Principles of emergency planning and management: Oxford University Press, New York, USA (2002)

11. D. Alexander. Towards the development of a standard in emergency planning. Disaster Prevention and Management,14,2, 158-175 (2005)

12. B. Rittichainuwat. Tourists' and tourism suppliers' perceptions toward crisis management on tsunami. Tourism Management, 34, 112-121 (2013)

13. S. Becken and K.F.D. Hughey, Linking tourism into emergency management structures to enhance disaster risk reduction. Tourism Management, 36,0, 77-85 (2013) 
14. B. Prideaux, E. Laws, and B. Faulkner, Events in Indonesia: exploring the limits to formal tourism trends forecasting methods in complex crisis situations. Tourism Management, 24,4, 475-487(2003)

15. R.W. Perry and M.K. Lindell, Preparedness for emergency response: guidelines for the emergency planning process. Disasters, 27,4,336-350 (2003)

16. R. Dynes. "Coming to terms with community disaster", in Quarantelli, E.L. (Ed.), what Is a Disaster? Perspectives on the Question, Routledge, London, Pp. 109-126 (1998)

17. D. Parker. The mismanagement of hazards. Hazard management and emergency planning: perspectives on Britain. London: James and James Science Publishers (1992)

18. Coppola, D.P., Introduction to International Disaster Management. (2010): Elsevier Science. (2010)

19. K.A. Stahura et al., Emergency planning and recovery for terror situations: an analysis with special reference to tourism. Worldwide Hospitality and Tourism Themes, 4,1, 48-58. (2012)

20. E.L. Quarantelli. A Selected Annotated Bibliography of Social Science Studies on Disasters. American Behavioral Scientist, 13,3, 452-456 (1970)

21. T. Drabek. Disaster responses within the tourist industry. International Journal of Mass Emergencies and Disasters, 13,1, 7-23 (1995)

22. M.C. Burritt. The road to recovery: a look at the lodging industry, post-September 11. Real Estate Issues, 26, 4,15-18 (2002)

23. S. Fink. Crisis Management: Planning for the Inevitable. New York, NY: American Management Association (1986)

24. V. Roberts. Flood Management: Bradford Paper. Disaster Prevention and Management, 3,2, 44 - 60 (1994)

25. B. Faulkner. Towards a framework for tourism disaster management. Tourism Management, 22,2, 135-147 (2001)

26. R.W. Riley and L.L. Love, The state of qualitative tourism research. Annals of Tourism Research, 27, 1,164-187 (2000)

27. A.H. Walle. Quantitative versus qualitative tourism research. Annals of Tourism Research, 24, 3, 524-536 (1997)

28. M. Sandelowski. Sample size in qualitative research. Research in nursing \& health, 18, 2, 179183. (1995) 